\title{
Cyclotides chemosensitize glioblastoma cells to
}

\section{temozolomide}

Samantha L. Gerlach, ${ }^{*} \uparrow$ Rachael A. Dunlop,$\ddagger$ James S. Metcalf,$\ddagger$ Sandra A. Banack,, Paul Alan Cox $\ddagger$

${ }^{\dagger}$ Dillard University, Department of Biology, New Orleans, Louisiana, 70122, USA; Email:

sgerlach@dillard.edu

\#Brain Chemistry Labs, Institute for Ethnomedicine, Box 3464, Jackson, Wyoming, 83001, USA

* Corresponding author:

sgerlach@dillard.edu; Tel. 504-4601339; ORCID ID 0000-0003-3398-1565 


\section{TABLE OF CONTENTS:}

Figure S1: The reference $\mathrm{CyO} 2$ displays a doubly charged ion $(\mathrm{M}+2 \mathrm{H})$ at $m / z, 1570.69 \ldots . . . . .$. page 3

Figure S2: Purified CyO2 from the crude extract of Viola odorata displays a doubly charged ion $(\mathrm{M}+2 \mathrm{H})$ at $m / z \mathbf{1 5 7 0 . 6 9}$ page 4

Figure S3: The reference CyO2 displays a doubly charged ion $(\mathrm{M}+2 \mathrm{H})$ at $m / z, 1753.77$, which, corresponds to the reduction and alkylation of 6 cysteine residues and the linearization of a cyclic cyclotide with endoproteinase Glu-C. page 5

Figure S4: The purified CyO2 from the crude extract of Viola odorata displays a doubly charged ion $(\mathrm{M}+2 \mathrm{H})$ at $m / z$ 1753.77, which corresponds to the reduction and alkylation of 6 cysteine residues and the linearization of a cyclic cyclotide with endoproteinase Glu-C......... page 6

Figure S5: Varv peptide A was not effective at sensitizing SH-SY5Y cells to TMZ..............pages 7 - 8

Figure S6: Titrations of $\mathrm{CyO2}$ and TMZ in U-87 MG cell cultures to determine the optimal co-exposure to reduce the concentration of $\mathrm{TMZ}$ required to induce $\mathrm{IC}_{50}$ 


\section{SUPPORTING INFORMATION}
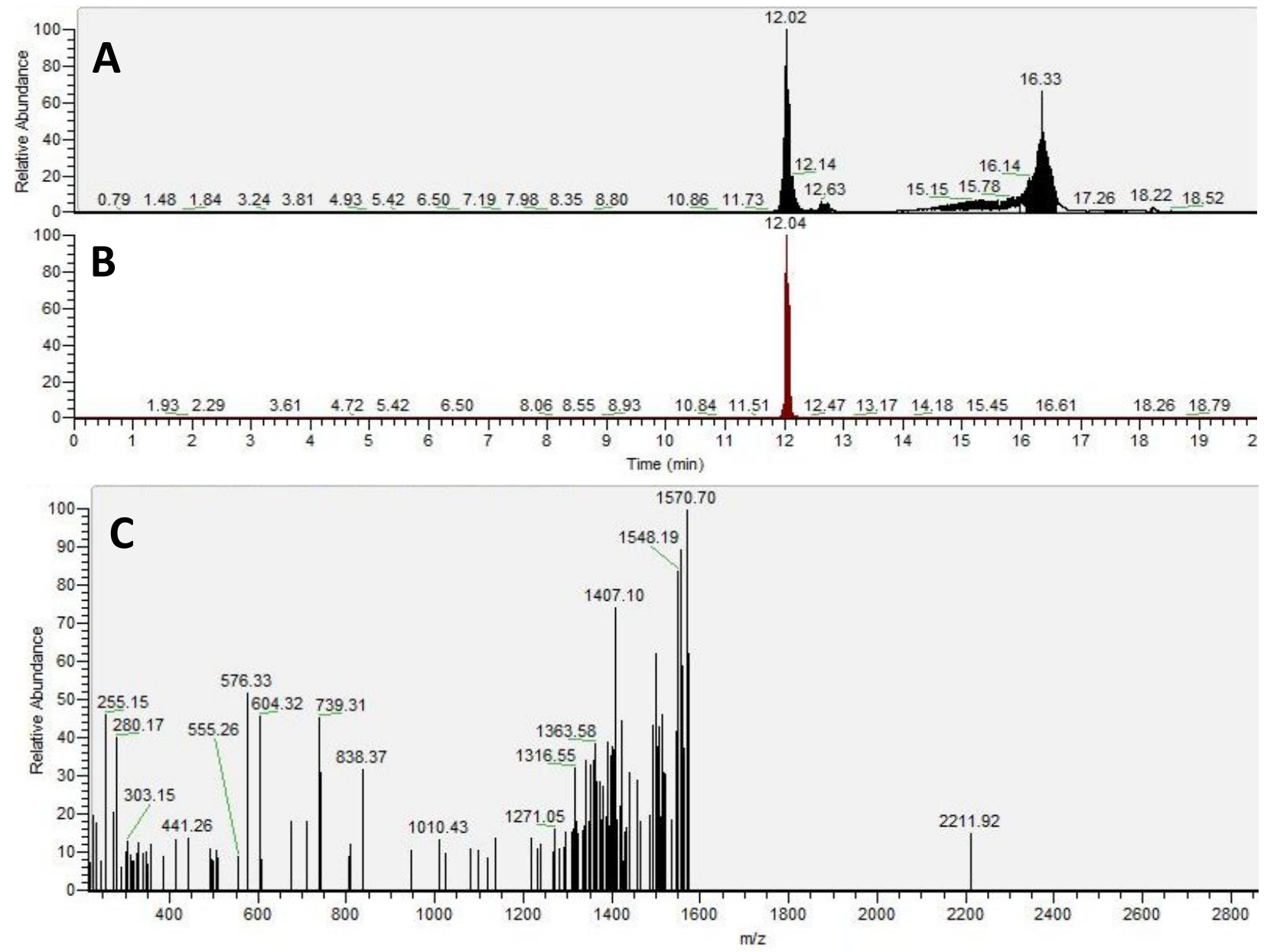

Figure S1. The reference CyO2 displays a doubly charged ion $(\mathrm{M}+2 \mathrm{H})$ at $\boldsymbol{m} / \boldsymbol{z}, \mathbf{1 5 7 0 . 6 9}$. Panel $\mathrm{A}$ is a total ion chromatogram (TIC) of reference $\mathrm{CyO} 2$, and Panel $\mathrm{B}$ is an extracted ion chromatogram (EIC) of doubly charged $\mathrm{CyO} 2$ at $m / z 1570.69$ for the reference $\mathrm{CyO} 2$. Panel $\mathrm{C}$ displays MS/MS data of daughter ions derived from a parent ion of $\mathrm{m} / \mathrm{z} 1570.69$ for the reference $\mathrm{CyO} 2$ at 12 minutes. 

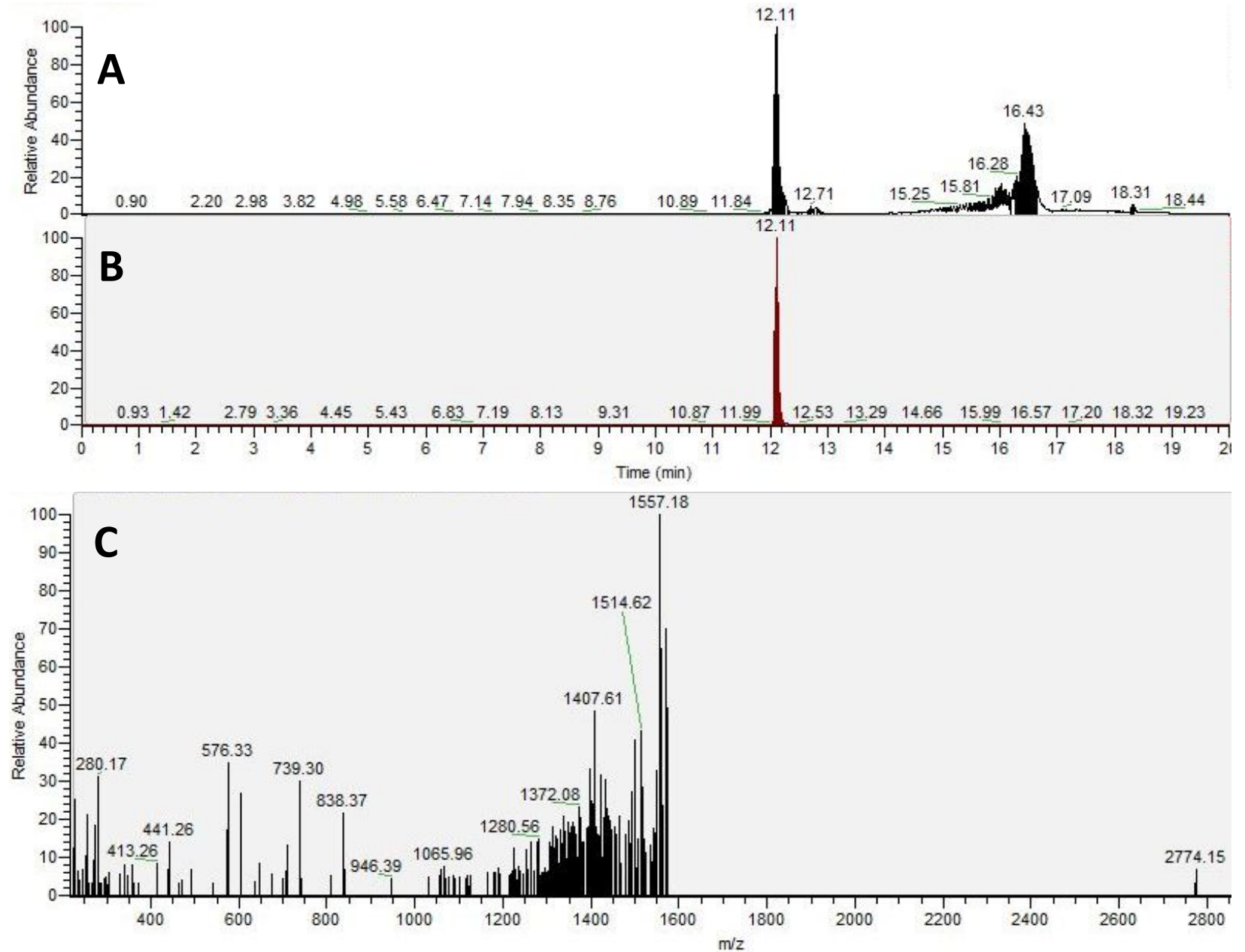

Figure S2. Purified CyO2 from the crude extract of Viola odorata displays a doubly charged ion $(\mathrm{M}+2 \mathrm{H})$ at $\boldsymbol{m} / \boldsymbol{z}$ 1570.69. Panel $\mathrm{A}$ is a total ion chromatogram (TIC) of the purified CyO2 from the crude extract of $V$. odorata, and Panel B is an extracted ion chromatogram (EIC) of doubly charged CyO2 at $\mathrm{m} / z 1570.69$ for the purified $\mathrm{CyO} 2$ from the crude extract of $V$. odorata. Panel $\mathrm{C}$ displays MS/MS data of daughter ions derived from a parent ion of $\mathrm{m} / \mathrm{z} 1570.69$ for the purified $\mathrm{CyO} 2$ from the crude extract of $V$. odorata at 12 minutes. 



Figure S3. The reference CyO2 displays a doubly charged ion $(\mathrm{M}+2 \mathrm{H})$ at $m / z, 1753.77$, which corresponds to the reduction and alkylation of 6 cysteine residues and the linearization of a cyclic cyclotide with endoproteinase Glu-C. Panel A is a total ion chromatogram (TIC) of treated reference CyO2, and Panel B is an extracted ion chromatogram (EIC) of doubly charged CyO2 at $m / z 1753.77$ for the modified reference CyO2. Panel C displays MS/MS data of daughter ions derived from a parent ion of $m / z 1753.77$ for the modified reference $\mathrm{CyO} 2$ at 10.7 minutes. The unmodified reference $\mathrm{CyO} 2$ for the doubly charged ion at $\mathrm{m} / z 1570.69$ plus $183.01 \mathrm{Da}$ equals the doubly charged ion at $\mathrm{m} / \mathrm{z} 1753.77$, which provides supporting evidence that the unmodified reference $\mathrm{CyO} 2$ was cyclic and contained 6 cysteine residues. 

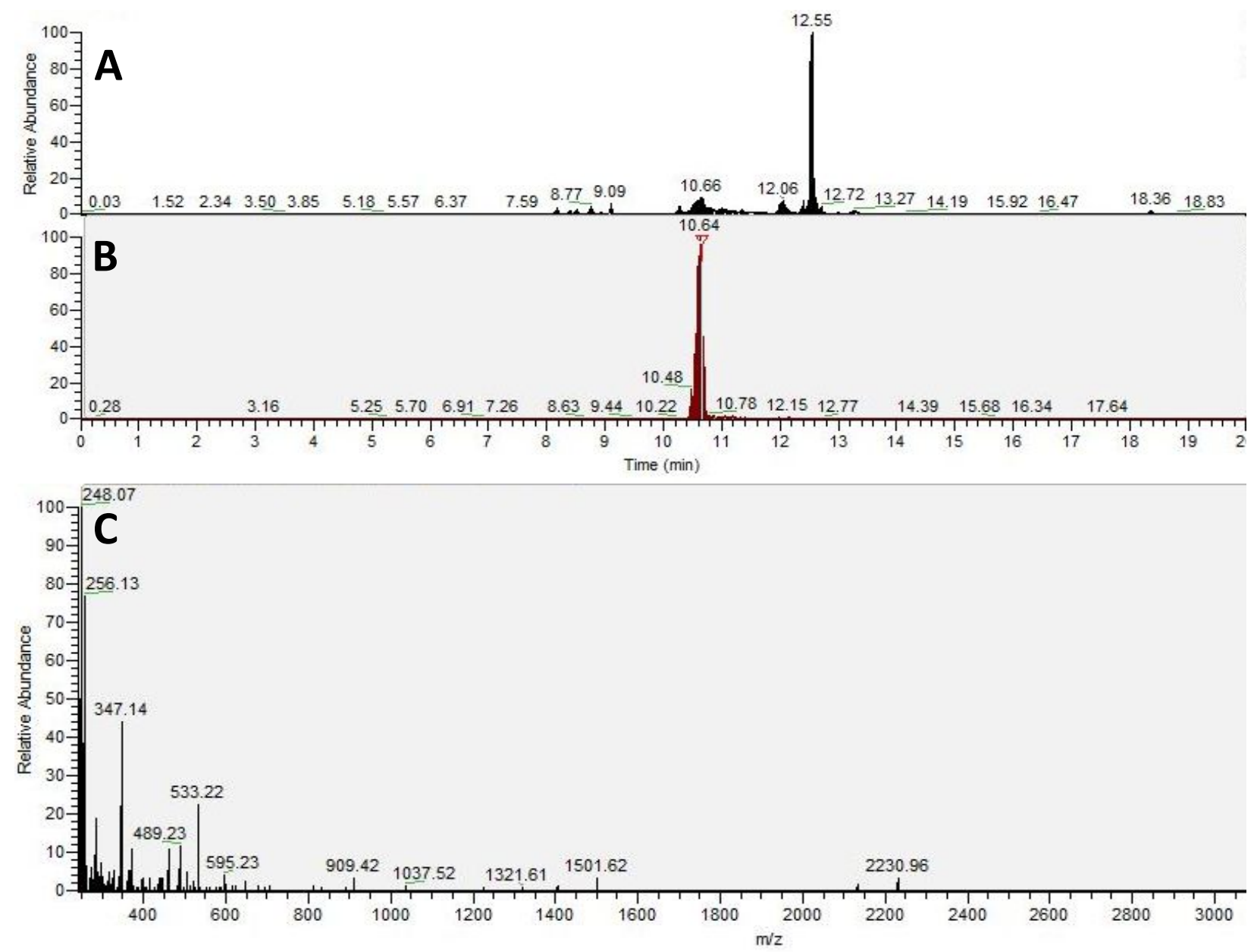

Figure S4. The purified $\mathrm{CyO} 2$ from the crude extract of Viola odorata displays a doubly charged ion $(\mathrm{M}+2 \mathrm{H})$ at $m / z$ 1753.77, which corresponds to the reduction and alkylation of 6 cysteine residues and the linearization of a cyclic cyclotide with endoproteinase Glu-C. Panel A is a total ion chromatogram (TIC) of the treated purified $\mathrm{CyO} 2$ from the crude extract of $V$. odorata, and Panel $\mathrm{B}$ is an extracted ion chromatogram (EIC) of doubly charged $\mathrm{CyO} 2$ at $\mathrm{m} / z 1753.77$ for the modified purified $\mathrm{CyO} 2$ from the crude extract of $V$. odorata. Panel C displays MS/MS data of daughter ions derived from a parent ion of $\mathrm{m} / z 1753.77$ for the modified purified $\mathrm{CyO} 2$ from the crude extract of $V$. odorata at 10.6 minutes. The unmodified purified $\mathrm{CyO} 2$ from the crude extract of $V$. odorata for the doubly charged ion at $m / z 1570.69$ plus 183.01 Da equals the doubly charged ion at $m / z$ 1753.77, which provides supporting evidence that the unmodified purified $\mathrm{CyO} 2$ from the crude extract of $V$. odorata was cyclic and contained 6 cysteine residues. 

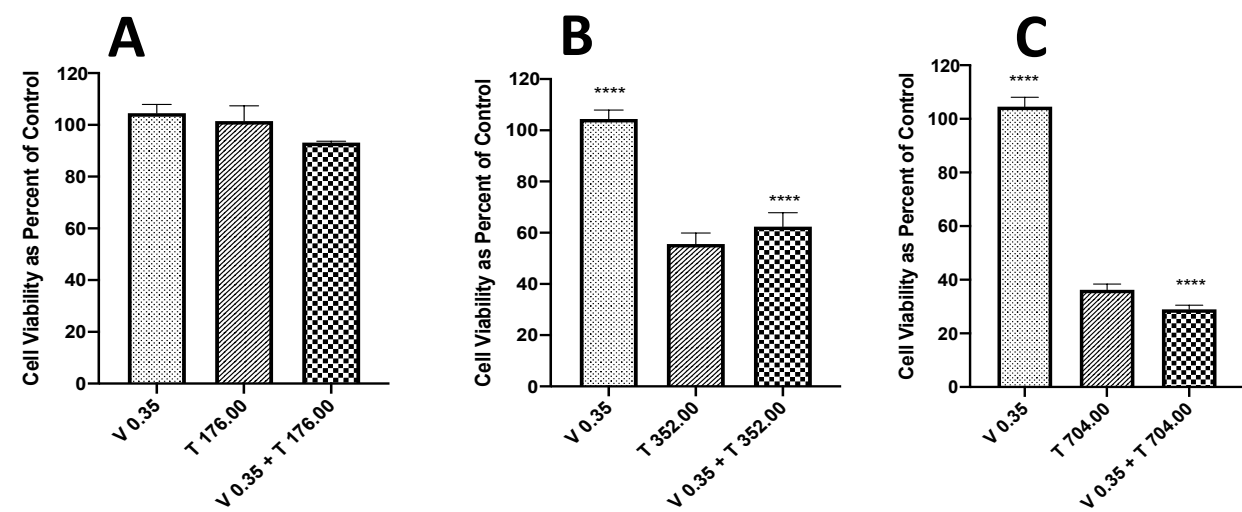

[Varv peptide A, TMZ, Co-exposure] $\mu \mathrm{M}$

[Varv peptide A, TMZ, Co-exposure] $\mu \mathrm{M}$

[Varv peptide A, TMZ, Co-exposure] $\mu \mathrm{M}$

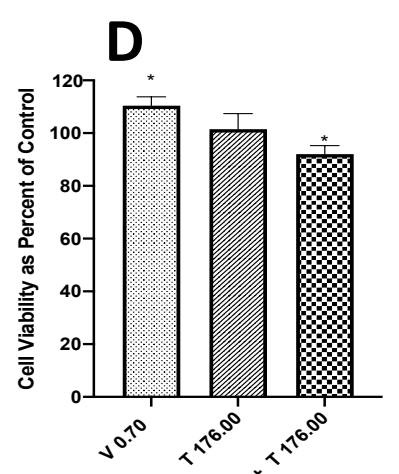

E
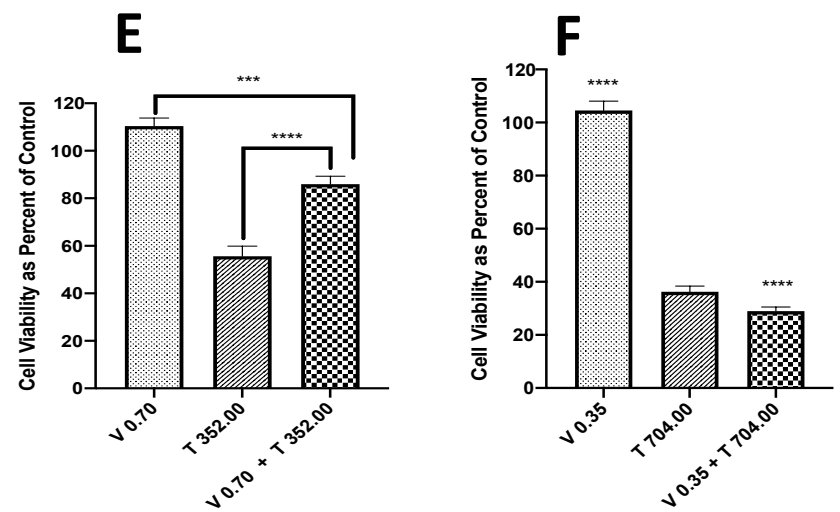

[Varv peptide A, TMZ, Co-exposure] $\mu \mathrm{M}$

[Varv peptide A, TMZ, Co-exposure] $\mu \mathrm{M}$

[Varv peptide A, TMZ, Co-exposure] $\mu \mathrm{M}$


[Varv peptide A, TMZ, Co-exposure] $\mu \mathrm{M}$

[Varv peptide A, TMZ, Co-exposure] $\mu \mathrm{M}$

[Varv peptide A, TMZ, Co-exposure] $\mu \mathrm{M}$


[Varv peptide A, TMZ, Co-exposure] $\mu \mathrm{M}$ 
Figure S5: Varv peptide A was not effective at sensitizing SH-SY5Y cells to TMZ. To determine if Varv peptide A (V) enhances TMZ (T) induced neuroblastoma cell death, MTT was measured after incubating cell cultures with concentrations of Varv peptide A (V $0.35-2.80 \mu \mathrm{M})$ and TMZ (T $176.00-704.00 \mu \mathrm{M})$ alone and co-exposed to Varv peptide A and TMZ. The first dotted bars in each panel indicate concentrations of Varv peptide A exposed to cells alone while the second diagonal stripe bars indicate TMZ exposed to cells alone. The third checkered bars indicate co-exposure treatments with Varv peptide A and TMZ. Cells were plated into 96well plates (3000 cells per well) and treated with cyclotides as indicated for 72 hours. MTT was measured using a kit from Roche (see materials and methods). Cell viability was converted to percentage and expressed as a percentage of control cultures (culture medium treated with DMSO only). Incubations were conducted in triplicate wells (technical replicates) and as 3 independent incubations (biological replicates, $\mathrm{n}=3$ ). Data is expressed as mean +/- standard error of the mean (SEM). Results of one-way ANOVA and Tukey's multiple comparisons are graphed in GraphPad Prism version 8 for iOS with the following p-values: $*<0.05, * *<0.01$, $* * *<0.001$ and $* * * *<0.0001$.

The experimentally determined $\mathrm{IC}_{50}$ Best Fit for Varv peptide A alone in SH-SY5Y cells was $2.88 \mu \mathrm{M}$ (Table 2). When we combined Varv peptide $\mathrm{A}(2.88 \mu \mathrm{M})$ with the $\mathrm{IC}_{50}$ Best Fit for TMZ $(393.00 \mu \mathrm{M})$ in SH-SY5Y cells, there was no significant difference between treatments. Further, the combination treatment of TMZ and Varv peptide A did not increase cell death (Figure S5, Panel K) above individual treatments alone. We tested a range of concentrations of Varv peptide $\mathrm{A}$ and $\mathrm{TMZ}$ and none of these reached significance between any conditions in this model. As demonstrated in Panels B, C, D, F, H, I and L, although co-exposures (V $0.35+\mathrm{T}$ 352.00, V 0.35 + T 704.00, V 0.70 + T 176.00, V 0.70 + T 704.00, V 1.40 + T 352.00, V 1.40 + T 704.00, and $\mathrm{V} 2.80+\mathrm{T} 704.00 \mu \mathrm{M}$ respectively) were significantly different compared to the cyclotide exposure alone (V $0.35, \mathrm{~V} 0.70, \mathrm{~V}, 1.40$, and $\mathrm{V} 2.80 \mu \mathrm{M}$ ), they were not significantly different when compared to their corresponding TMZ concentrations alone (T 176.00, T 352.00 and T 704.00 $\mu \mathrm{M}$ ). Contrastingly, Panel J indicates that co-exposure of Varv peptide $\mathrm{A}(2.80 \mu \mathrm{M})$ plus TMZ $(176.00 \mu \mathrm{M})$ had a significant reduction in cell viability compared to TMZ alone; however, there was no significance when the co-exposure was compared to Varv peptide A alone. This suggests that Varv peptide A in combination with TMZ is not effective at sensitizing cells to TMZ cytotoxicity in SH-SY5Y cell from $\sim 110 \%$ at Varv peptide A $(1.40 \mu \mathrm{M}), 100 \%$ for TMZ $(176.00 \mu \mathrm{M})$ and $80 \%$ for combined Varv peptide A and TMZ. 


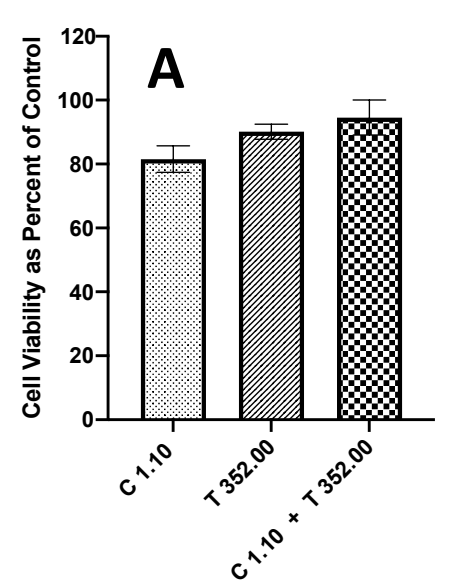

[CyO2, TMZ, Co-exposure] $\mu M$

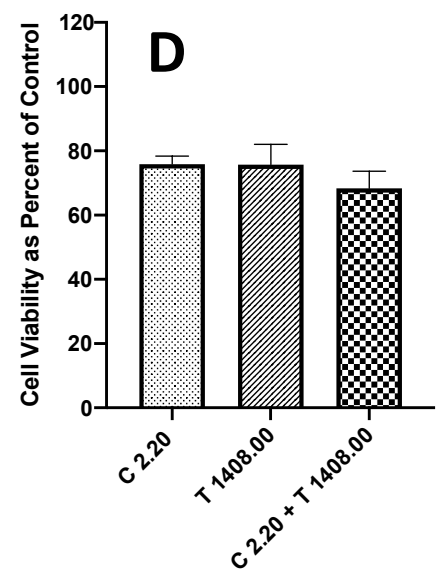

[CyO2, TMZ, Co-exposure] $\mu \mathrm{M}$

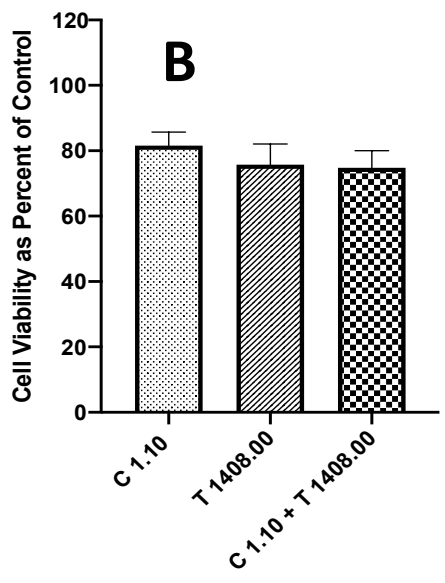

[CyO2, TMZ, Co-exposure] $\mu \mathrm{M}$



[CyO2, TMZ, Co-exposure] $\mu \mathrm{M}$



[CyO2, TMZ, Co-exposure] $\mu \mathrm{M}$

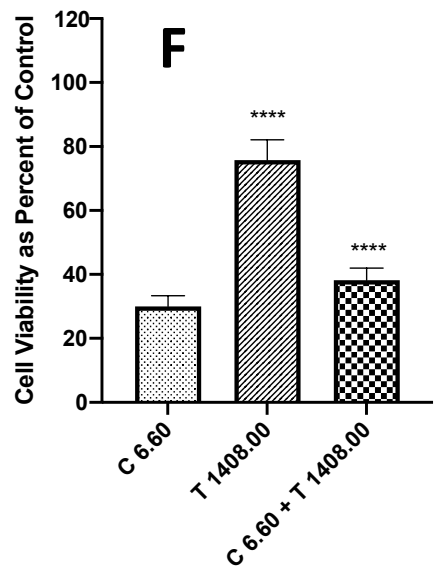

[CyO2, TMZ, Co-exposure] $\mu \mathrm{M}$

Figure S6: Titrations of $\mathrm{CyO2}$ and $\mathrm{TMZ}$ in U-87 MG cell cultures to determine the optimal co-exposure to reduce the concentration of $\mathbf{T M Z}$ required to induce $\mathbf{I C}_{\mathbf{5 0}}$. To determine if $\mathrm{CyO} 2(\mathrm{C})$ enhances $\mathrm{TMZ}(\mathrm{T})$ induced glioblastoma cell death, we measured MTT after incubating cell cultures with concentrations of CyO2 $(0.27-2.15 \mu \mathrm{M})$ and TMZ $(54.05-216.20 \mu \mathrm{M})$ alone and co-exposed to CyO2 and TMZ. The first dotted bars in each panel indicate cells exposed to concentrations of $\mathrm{CyO} 2$ alone while the second diagonal stripes indicate cells exposed to TMZ alone. The third checkered bars indicate co-exposure treatments with CyO2 and TMZ. Cells were plated into 96-well plates (3000 cells/well) and treated as indicated for 72 hrs. Cell viability was measured using (see materials and methods). Cell viability is expressed as a percentage of control cultures (culture medium with DMSO only). Incubations were conducted in triplicate wells (technical replicates) and as 3 independent incubations (biological replicates, $n=3$ ). Data is expressed as mean $+/$ - standard error of the mean (SEM). Results of one-way ANOVA and Tukey's multiple comparisons are graphed in GraphPad Prism version 8 for iOS with the following p-values: $*<0.05, * *<0.01, * * *<0.001$ and $* * * *<0.0001$.

As shown in Panel C, there was a significant difference between TMZ treatment alone $(352.00 \mu \mathrm{M})$ and coexposure treatment $(\mathrm{C} 2.20+\mathrm{TMZ} 352.00 \mu \mathrm{M})$; however, this significance did not persist when comparing coexposure to $\mathrm{CyO} 2$ alone. Panels $\mathrm{E}$ and $\mathrm{F}$ demonstrate that co-exposure of cells to $\mathrm{CyO} 2$ and TMZ $(\mathrm{C} 6.60+\mathrm{T}$ 352.00 and C $6.60+\mathrm{T} 1408.00)$ showed significantly reduced cell viability when compared to TMZ alone (Panel G: $352.00 \mu \mathrm{M}$ and Panel H: $1408.00 \mu \mathrm{M}$ ), but there was no significant difference when comparing cell viability of $\mathrm{CyO} 2$ alone $(6.60 \mu \mathrm{M})$ to co-exposures. 